\title{
Building Hybrid Automata of Complex Physical Systems for Real-time Applications
}

\author{
Pieter J. Mosterman ${ }^{1}$ \\ Institute of Robotics and System Dynamics \\ DLR Oberpfaffenhofen \\ D-82230 Wessling \\ Pieter.J.Mosterman@dIr.de
}

\author{
Gautam Biswas ${ }^{2}$ \\ Dept. of Electrical Engineering and Computer Science \\ Vanderbilt University \\ Nashville, TN 37235 \\ bistras@vuse. vanderbilt . edu
}

\begin{abstract}
Dynamic behavior of complex physical systems is often nonlinear and includes multiple temporal scales. Singular perturbation methods decouple the fast and slow behavior and by assurning that the fast behavior is at a quasi steady state, the slow behavior of the system can be analyzed. The decoupling reduces the complex system of ordinary differential equations (ODEs) to simpler ODEs that allow fixed time step integration methods, and, therefore, are suitable for real-time applications. This model reduction may cause discontinuous jumps in the initial values of model variables. This paper applies and extends singular perturbation methods to compute discontinuous state changes for piecewise continuous, hybrid, models when the model configuration changes. Computing the explicit state change allows the use of hybrid automata as modeling framework when augmented with execution semantics for state vector updates around discontinuities.
\end{abstract}

\section{Introduction}

The pressure to achieve optimal performance and meet rigorous safety standards in industrial processes, aircraft, and nuclear plants, necessitates more detailed modeling and analysis of these systems. Complex systems exhibit nonlinearities attributed to small parameters that manifest as behaviors on very fast time scales that complicate this task. In case of numerical simulation, sophisticated algorithms vary their time step to accommodate multi time scale behaviors, but the variable step size makes it hard to bound their runtime computational complexity. This makes them unsuitable for real-time applications. As an alternative, $h y$ brid nodeling methodologies represent system behavior as piecewise continuous modes interspersed with discrete transitions. This facilitates the use of fixed time

\footnotetext{
${ }^{1}$ Pieter J. Mosterman is supported by a grant from the DFG Schwerpunktprogramm KONDISK.

2 Gautam Biswas is supported by grants from HewlettPackard, Co.
}

step integration methods to numerically simulate the continuous dynamics, whereas the fast dynamics are replaced by instantaneous changes, allowing real-time simulation.

The primary flight control system of aircraft (illustrated in Fig. 1) demonstrates the paradigm for hybrid, i.e., mixed continuous/discrete, modeling of embedded control systems. At the lowest level in the control hierarchy, continuous PID control noves the rudder, elevators, and ailerons to desired set positions. On a higher level, digital control may mandate mode changes at different stages of a flight plan (e.g., take-off, cruise, go-around) and detection of failures may lead to discrete changes in system configuration. Furthermore, abstracting fast, nonlinear transients may produce discontinuous variable changes.

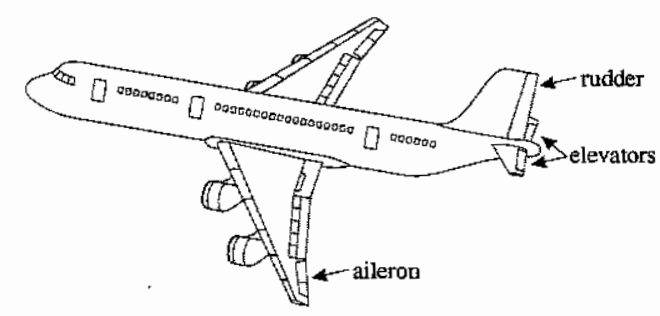

Figure 1: Primary aerodynamic control surfaces.

The singular perturbation approach [4] abstracts detailed continuous behavior represented as a system of complex nonlinear ordinary differential equations, cODE, into piecewise simpler sODEs. Our goal is to extend and generalize this approach to apply to hybrid systems that include one or a number of, possibly consecutive, discrete mode changes. We have shown $[6,8]$ that small time constant effects cannot always be removed (parameter abstractions) in analyzing dynamic system behavior. Abstracting fast transients may require explicit additional jump constraints for the system state vector variable values when configuration changes occur (time scale abstraction).

This paper presents the effects of abstracting fast continuous transients exhibited by complex systems into 
discontinuous changes of the continuous state vector. It demonstrates a systematic methodology for generating the simpler ODE models from the more complex ODE models of system behavior. The simpler piecewise ODE models are then compiled into hybrid automata [1] with extended execution semantics to facilitate efficient real-time applications.

\section{Hybrid Dynamic Systems}

Hybrid dynamic systems combine discrete state changes with continuous behavior evolution $[1,5,8]$. Differential equations form a common representation of continuous system behavior. The system is described by a state vector, $x$. Behavior over time is specified by a field $f$. Interaction with the environment is specified by input and output signals, $u$ and $y$. The dynamics of system behavior is expressed as a set of ODEs, $\dot{x}=f(x, u)$ and algebraic relations $y=h(x)$.

Discrete behavior can be modeled by a state machine representation, consisting of a set of discrete modes, $\alpha$. Mode changes caused by events, $\sigma$, are specified by the state transition function $\phi$, i.e., $\alpha_{i+1}=\phi\left(\alpha_{i}\right)$. A transition may produce additional discrete events, causing further transitions.

A mode change from $\alpha_{i}$ to $\alpha_{i+1}$, may result in a field definition change from $f_{\alpha_{i}}$ to $f_{\alpha_{i+1}}$, and a discontinuous change in the state vector governed by an algebraic function $g, x^{+}=g_{\alpha_{i}}^{\alpha_{i+1}}(x)$. Discrete mode changes are caused by an event generation function, $\gamma$, associated with the current active mode, $\alpha_{i}, \gamma_{\alpha_{i}}(x) \leq 0 \rightarrow \sigma_{j}$.

\section{Abstracting Fast Transients}

Continuous behavior in physical systems can occur on a hierarchy of temporal and spatial scales. To simplify system models, small ${ }^{1}$ parameters are abstracted away but this may cause discontinuous changes in system behavior. These discontinuous changes are present as fast continuous transients in the more detailed models. In this section, we formalize the derivation of simphified models generated by parameter and time scale abstractions and derive explicit discontinuous state changes to facilitate the use of the simpler models in a hybrid autoinata framework.

\subsection{Parameter Abstraction}

Parameter abstractions elimmate small parameters in a system model. A singular perturbation representation formulates the system behavior model into a cODE

\footnotetext{
${ }^{1}$ Also, large, because the reciprocal of a large parameter value is small.
}

with two time scales:

$$
\left\{\begin{array}{l}
\dot{x}=f(x, z, \epsilon, t), x\left(t_{0}\right)=x^{0}, x \in \Re^{n}, \\
\epsilon \dot{z}=g(x, z, \epsilon, t), z\left(t_{0}\right)=z^{0}, x \in \Re^{m},
\end{array}\right.
$$

where $\epsilon$ is a small parameter that embodies small and large parameter values that cause fast transients. The function $f$ models the slower dominant system behavior. Setting $\epsilon$ to 0 reduces the second equation to an algebraic form. Assuming that $g(x, z, 0, t)=0$ has distinct real roots, the fast behaviors corresponding to $z$ can be solved for algebraically, and substituted in $f$. This results in a reduced-order quasi steady state model that embodies an sODE.

We apply this approach to the collision between two bodies shown in Fig 2. A first order approximation of the collision process includes two parameters: (i) $C$, that models the elastic interaction between the bodies, and (ii) $R$, that models the dissipative effects. If the momentum, $p_{i}$, of the bodies, $m_{i}$, and the displacement, $q$, of the spring that models the elasticity parameter $C$, are chosen as state variables, the dynamic behavior of the system is described by the following ODE:

$$
\left\{\begin{array}{l}
\dot{p}_{1}=-\frac{q}{C}-R\left(\frac{p_{1}}{m_{1}}-\frac{p_{2}}{m_{2}}\right) \\
\dot{p}_{2}=\frac{q}{C}+R\left(\frac{p_{1}}{m_{1}}-\frac{p_{2}}{m_{2}}\right) \\
\dot{q}=\frac{p_{1}}{m_{1}}-\frac{p_{2}}{m_{2}}
\end{array}\right.
$$

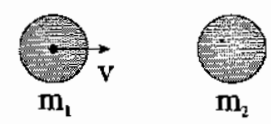

Figure 2: Collision of a body $m_{1}$ and a body $m_{2}$.

This singular system of equations can be reduced by applying the transformation $v=\frac{p_{1}}{m_{1}}-\frac{p_{2}}{m_{2}}$, resulting $\mathrm{m}$ a second order ODE

$$
\left[\begin{array}{c}
\dot{v} \\
\dot{q}
\end{array}\right]=\left[\begin{array}{cc}
-R\left(\frac{1}{m_{1}}+\frac{1}{m_{2}}\right) & -\frac{1}{C}\left(\frac{1}{m_{1}}+\frac{1}{m_{2}}\right) \\
1 & 0
\end{array}\right]\left[\begin{array}{l}
v \\
q
\end{array}\right] \text {. }
$$

If the $R$ and $C$ parameters are removed from the model, a simpler system of equations results, but the state variables may exhibit explicit discontinuous jumps. The hybrid automata model requires explicit definition of these jumps, and necessitates their computation from the detailed continuous transients.

To apply singular perturbations, we assume $C$ to be small and $R$ to be large and take $\frac{1}{R}$ to be the small $\epsilon$ parameter. Therefore,

$$
\left[\begin{array}{c}
\frac{1}{R} \dot{v} \\
\dot{q}
\end{array}\right]=\left[\begin{array}{cc}
-\left(\frac{1}{m_{1}}+\frac{1}{m_{2}}\right) & -\frac{1}{R C}\left(\frac{1}{m_{1}}+\frac{1}{m_{2}}\right) \\
1 & 0
\end{array}\right]\left[\begin{array}{l}
v \\
q
\end{array}\right]
$$

where $v$ contains the fast behavior. Substituting $\frac{1}{R}=0$ results in $v=0$. Transforming this back to the original state variables yields $\frac{p_{1}}{m_{1}}-\frac{p_{2}}{m_{2}}=0$, i.e., $v_{1}-v_{2}=0$. This is the equivalent of a perfect non elastic collision [6]. 


\subsection{Time Scale Abstraction}

Instead of eliminating the fast transient due to dissipative effects, if we were to reduce the effect of elasticity to occur at a point in time, we get a time scale abstraction. Consider the system of colliding bodies again (Fig. 2) with detailed behavior given by Eq. (3). If $C$ is taken to be the small $\epsilon$ parameter, this gives

$$
\left[\begin{array}{c}
C \dot{v} \\
\dot{q}
\end{array}\right]=\left[\begin{array}{cc}
-R C\left(\frac{1}{m_{1}}+\frac{1}{m_{2}}\right) & \left(\frac{1}{m_{1}}+\frac{1}{m_{2}}\right) \\
1 & 0
\end{array}\right]\left[\begin{array}{l}
v \\
q
\end{array}\right],
$$

For $\epsilon=0$, this yields $q=0$, and, therefore, $\dot{q}=0$ which requires $v=0$. When $C$ becomes small but not 0 , the solution of the system in Eq. (3) has eigenvalues with imaginary components and the resultant dynamic behavior for the transient is:

$v(t)=v(0) e^{-\frac{R}{2}\left(\frac{1}{m_{1}}+\frac{1}{m_{2}}\right) t} \cos \left(\left(\sqrt{\frac{4}{C}-R^{2}\left(\frac{1}{m_{1}}+\frac{1}{m_{2}}\right)^{2}}\right) t\right)$.

This shows that $v=0$ is the steady state solution. However, in case of colliding bodies this behavior transient is aborted long before steady state is attained, because the $v$ and $q$ values generated by the transient cause the two bodies to disconnect.

This is illustrated by the case of two point masses that collide when $x_{1} \geq x_{2}$, where $x_{1}$ and $x_{2}$ are the positions of body $m_{1}$ and $m_{2}$, respectively. The bodies disconnect when the force between them becomes negative, i.e., $F_{12}<0$. At this point, the state variable values (i.e., the two body velocities) constitute the final, $a$ posteriori, values around the discontinuous jump corresponding to the collision. Since $F_{12}=\frac{q}{C}<0$ at the disconnect point, this implies $q<0$ since $C>0$. The time point at which the disconnect occurs is computed to be

$$
t_{d}=\frac{\pi}{\sqrt{\frac{4}{C}-R^{2}\left(\frac{1}{m_{1}}+\frac{1}{m_{2}}\right)}} .
$$

At $t_{d}, v$ has changed from $v(0)$ to $v\left(t_{d}\right)=\lambda v(0)$ with $(\cos (\pi)=-1)$, therefore,

$$
\lambda=-e^{-\frac{R}{2}\left(\frac{1}{m_{1}}+\frac{1}{m_{2}}\right) t_{\delta}}
$$

As the $C$ parameter becomes very small, $t_{d}$ does too, and in the limit, $v\left(t_{d}\right) \rightarrow v(0)^{+}$. The discontinuous change in $v$ can then be represented by an algebraic equation $v(0)^{+}=\lambda v(0)$. Transforming this back to the original state variables yields $\frac{p_{1}^{+}}{m_{1}}-\frac{p_{2}^{+}}{m_{2}}=\lambda\left(\frac{p_{1}}{m_{1}}-\frac{p_{2}}{m_{2}}\right)$. Written in terms of the body velocities,

$$
v_{1}^{+}-v_{2}^{+}=\lambda\left(v_{1}-v_{2}\right)
$$

this is the well known Newton's collision rule [2], where $\lambda$ is called the coefficient of restitution that describes the amount of kinetic energy loss in the collision. If $R=0$ in Eq. (8), $\lambda=-1$ and this describes a perfect elastic collision with no loss of energy. Note that $C$ cannot be taken to equal 0 , as this would remove all elasticity and the corresponding ideal rigid body collision has no mechanism for storing kinetic energy as potential energy and returning it as kinetic energy. Therefore, this immediately causes $v=0$. Consequently, behavior does not converge uniformiy as $C \rightarrow 0$.

\section{The Elevator System}

The elevator control subsystem in Fig. 1 consists of two mechanical elevators that are positioned by two electrohydraulic actuators each $[3,9]$. When a failure occurs, redundancy management may switch actuators to ensure maximum control.

Figure 3 shows the operation of one actuator. The continuous PID control mechanism for elevator positioning is implemented by a servo valve. The output of the servo valve controls the direction and speed of travel of the piston in the cylinder. A spool valve mechanism controls whether the actuator is active or passive. The piston in the positioning cylinder and connected elevator flap constitute the load and excessive pressures are prevented by a pressure rehief valve.

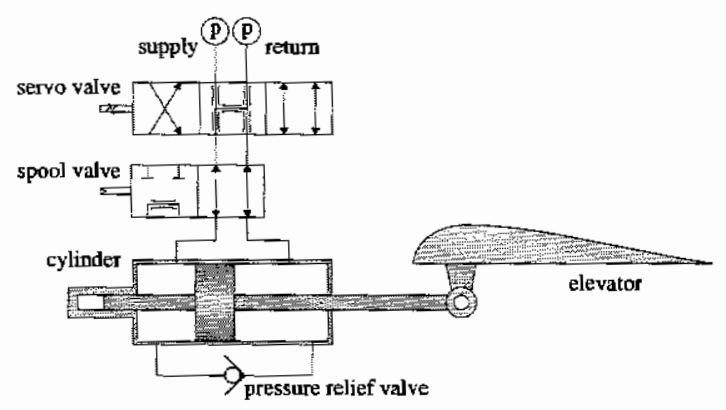

Figure 3: Hydraulics of one actuator.

\subsection{The Components}

The servo valve consists of a cylinder that connects its supply and return side with its loading side. A piston inside the cylinder controls the amount of oil flow from supply to loading. The amount of oil flowing in, $q_{s}$, has to equal the amount of oil flowing out $q_{t}$. This oil flow is determined by the pressure drop, $p_{s}-p_{l}$, across the orifice that is opened by an amount $x$, the PID control variable,

$$
\left\{\begin{array}{l}
q_{s}=\left(p_{s}-p_{l}\right) x \\
q_{s}=q_{l}
\end{array}\right.
$$

Like a servo valve, a typical spool valve (Fig. 4) consists of a piston that noves in a cylinder. A number of cylinder ports connect the supply and return part of the hydraulic system with the load. When the actuator is active, the spool valve is in its supply mode, 
$\alpha_{2}$, shown in Fig. 4(a), and the control signal generated by the servo valve is transferred to the cylinder that positions the elevator. In this mode, the pressure on the supply side of the valve, $p_{s}$, equals the pressure on the load side, $p_{t}$. Also, the oil flow from the supply, $q_{s}$, equals the oil flow to the load, $q_{l}$. When the actuator is passive, the spool valve is in its loading mode, $\alpha_{0}$, shown in Fig. $4(\mathrm{c})$, and control signals cannot be transferred to the cylinder. However, oil flow between the chambers is possible through a loading passageway with fluid flow resistance $R_{l}$. When moving between supply and loading, the spool valve passes through the closed configuration, $\alpha_{1}$, where oil flow is blocked, as shown in Fig. 4(b). This is captured by the following equations:

$$
\alpha_{2}:\left\{\begin{array}{c}
p_{s}=p_{l} \\
q_{s}=q_{l}
\end{array} \quad \alpha_{1}:\left\{\begin{array}{l}
q_{l}=0 \\
q_{s}=0
\end{array} \quad \alpha_{0}:\left\{\begin{array}{l}
p_{l}=q_{l} R_{l} \\
q_{s}=0
\end{array}\right.\right.\right.
$$

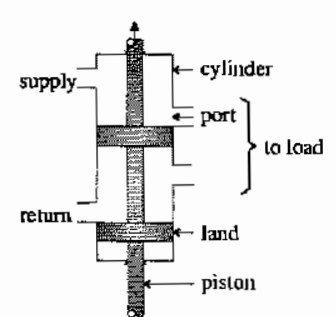

(a)

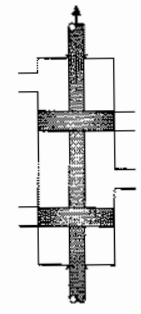

(b)

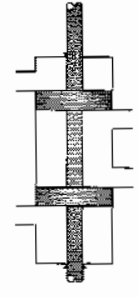

(c)
Figure 4: A typical spool valve.

A pressure relief valve connected to the positioning cylinder as a safety device is normally closed $\left(\alpha_{0}\right)$, but it may open $\left(\alpha_{1}\right)$ when the pressure in the elevator positioning cylinder, i.e., the input pressure to the relief valve, $p_{r}$, exceeds a threshold value, $p_{t h}$. When open, it allows an oil flow, $q_{r}$, through a fluid path with resistance $R_{l}$ :

$$
\alpha_{0}:\left\{q_{r}=0 \quad \alpha_{1}:\left\{p_{r}=q_{r} R_{l}\right.\right.
$$

\subsection{Modeling the Elevator Dynamics}

The dynamics of the elevator are studied in terms of the movement of the piston in the positioning cylinder. The behavior can be derived by composing models of the servo valve, spool valve, relief valve, and the positioning cylinder. This results in a second order system with two state variables: (i) $p_{c}$, the pressure of the oil in the cylinder, and (ii) $v_{e}$, the elevator velocity.

$$
\left\{\begin{array}{l}
C_{c} \dot{p}_{c}=q_{i n}+q_{r}-q_{e} \\
q_{e}=A_{p} v_{e} \\
A_{p} F_{e}=p_{c}+R_{c}\left(q_{i n}+q_{r}-q_{e}\right) \\
m_{e} \dot{v}_{e}=F_{e}
\end{array}\right.
$$

$C_{e}$ models the elasticity effects and $R_{c}$ models the dissipative effects of the oil in the positioning cylinder. The variables $q_{i n}$ and $q_{r}$ represent the inflow of oil into the cylinder from the servo and rehef valves, respectively, and $q_{e}$ represents the oil flow due to movement of the piston. The value of $q_{e}$ is a function of $A_{p}$, the area of the piston and $v_{e}$, the elevator velocity. The force exerted on the piston is a function of $p_{c}$, and the product of internal dissipation of the oil, $R_{c}$, and the overall flow rate. Newton's Second Law relates the elevator velocity to the force exerted on the piston. In state equation form $\mathrm{Eq} .(13)$ is:

$$
\begin{aligned}
{\left[\begin{array}{c}
\dot{p_{c}} \\
\dot{v_{e}}
\end{array}\right]=} & {\left[\begin{array}{cc}
0 & -\frac{A_{p}}{C_{c}} \\
\frac{1}{m_{e} A_{p}} & -\frac{R_{c}}{m_{e}}
\end{array}\right]\left[\begin{array}{l}
p_{c} \\
v_{e}
\end{array}\right] } \\
& +\left[\begin{array}{cc}
\frac{1}{C_{c}} & \frac{1}{C_{c}} \\
\frac{R_{c}}{m_{e} A_{p}} & \frac{R_{c}}{m_{e} A_{p}}
\end{array}\right]\left[\begin{array}{c}
q_{i n} \\
q_{r}
\end{array}\right]
\end{aligned}
$$

When a sudden pressure drop is detected in the hydraulics supply system of an elevator actuator, redundancy control moves the spool valve of this actuator from supply to loading and the spool valve of another actuator from loading to supply to take over control. When the spool valve of an actuator moves to its closed mode, oil flow into and out of the positioning cylinder is blocked. This implies that the cylimder piston that controls the elevator position cannot move, and the elevator stops moving as well. In more detail, the internal dissipation and small elasticity parameters of the oil cause the elevator velocity to change continuously during the transition. The continuous transient behavior between supply and closed is shown in Fig. 5(a). How quickly the system reaches 0 velocity iu the closed mode depends on the elasticity and internal dissipation parameters of the oil. Typically, soon after the closed mode, the spool valve starts opening and goes into the loading mode. The effect on the elevator velocity for the detailed continuous behavior wheu switching from supply to loading is shown in Fig. 5(b). A detailed analysis is presented in [7].

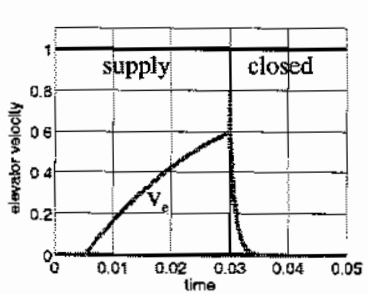

(a)

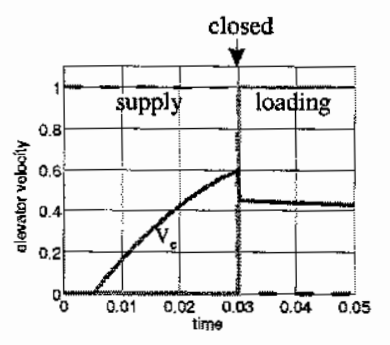

(b)
Figure 5: Continuous transients.

The details of the continuous transients are not of much interest for analysis of the control behavior. Model simplification by parameter and time scale abstractions results in removal of small elasticity and large dissipative effects. At the same time, the fast continuous transients that affect the elevator velocity, $v_{e}$, need to be preserved and captured by discontinuous changes. These 
discontinuities indicate that state variable dependencies arise between the model component ODEs. This requires model manipulations to generate a global ODE or methods to handle the resulting high index differential and algebraic equations (DAE).

We systematically derive the simpler models for the hybrid automata and the transition conditions using the methods based on singular perturbation described in Section 3 and replace the detailed continuous transients defined by Eq. (14) by an equation that captures the fast continuous change as an instantaneous discontinuous jump. We analyze the transient about the point where the spool valve closes, and the relief valve is also closed, i.e., $q_{\text {in }}=q_{r}=0$.

In case of real eigenvalues, the elevator dynamics can be computed to be $\left(D=\frac{R_{\varepsilon}^{2}}{m_{e}^{2}}-\frac{4}{m_{e} C_{c}}\right)$

$$
v_{e}(t)=e^{-\frac{R_{c}}{2 m_{e}} t}\left(k_{1} e^{\frac{1}{2}(\sqrt{D}) t}+k_{2} e^{-\frac{1}{2}(\sqrt{D}) t}\right),
$$

where $k_{1}$ and $k_{2}$ are constants that depend on $v_{e}(0)$ and $p_{c}(0)$. Like before, the restitution coefficient for the oil, affected by the spool valve closing, i.e., $\lambda_{s}$, can be computed by determining the value of $t_{d}$ at the point when the ports are opened again. If $x$ is the displacement of the piston in the spool valve, the piston may first block the ports when $x=0$ and open thein again when $x>x_{t h}$, where $x_{t h}$ is a parameter depending on the particular type of spool valve. The value of $t_{d}$ is then determined by $x_{t h}$ and the speed with which the piston is moved by an external control signal. The corresponding time interval during which the oil flow into the cylinder is 0 results in an elevator velocity change as a function of $v_{e}(0)$ and $p_{c}(0)$.

In case of complex eigenvalues, the elevator dynamic behavior is governed by

$$
v_{e}(t)=e^{-\frac{R_{c}}{2 m_{e}} t}\left(k_{1} \cos \left(\frac{1}{2}(\sqrt{-D}) t\right)+k_{2} \sin \left(\frac{1}{2}(\sqrt{-D}) t\right)\right)
$$

where $k_{1}$ and $k_{2}$ are constants depending on $v_{e}(0)$ and $p_{c}(0)$. Again, the change of elevator velocity at $t_{d}$ can be computed as a function of $v_{e}(0)$ and $p_{c}(0)$. In this case, the elevator velocity may reverse much like the velocity of a bouncing ball reverses.

\subsection{A Scenario}

Figure 6 defines the individual hybrid automata for the spool valve, the positioning cylinder, and the relief valve. When the spool valve goes from supply mode $\left(\alpha_{2}\right)$ to closed mode $\left(\alpha_{1}\right)$, causing $q_{s}$, and, therefore, $q_{i n}$, in the positioning cylinder to change discontinuously, the fast transient that affects $v_{e}$ can be simplified by parameter and time scale abstraction, and $v_{e}$ goes through an instantaneous change in velocity given by $v_{e}{ }^{+}=\lambda_{s} v_{e}$.

To compute the reduced continuous behavior model, the oil is assumed to be incompressible, the corresponding simplified ODE for elevator velocity in the positioning cylinder is calculated by setting $C_{c}=0$ :

$$
\left\{\begin{array}{l}
0=q_{i n}+q_{r}-q_{e} \\
q_{e}=A_{p} v_{e} \\
A_{p} F_{e}=p_{c} \\
m_{e} \dot{v}_{e}=F_{e}
\end{array}\right.
$$

This sODE is first order, whereas the cODE was second order.

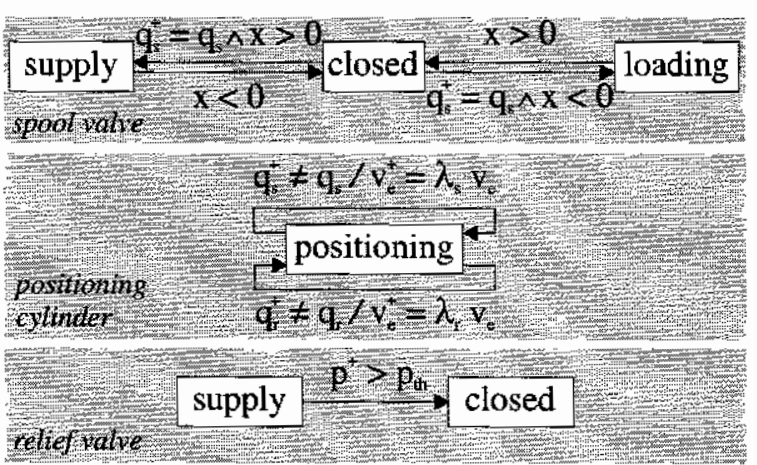

Figure 6: Individual hybrid automata.

In this model, because the behavior of the spool valve around $x=0$ is abstracted away; the spool valve switches into its closed mode when the piston in the valve reaches 0 from the right, $x<0$, or from the left, $x>0$. Now, $q_{s}^{+}=0$ and $q_{s}^{+} \neq q_{s}$, causing a discontinuous change in $v_{e}^{+}$. Immediately after the discontinuous changes are effected, $q_{s}^{+}=q_{s}$, and the spool valve switches out of the closed mode. Note that the abrupt change in velocity from $v_{e}$ to $v_{e}{ }^{+}$will cause a fast pressure buildup. In the reduced order model, this buildup is governed by a discontinuous change of $v_{e}$, and, therefore, $v_{e}^{+} \neq v_{e}$. The $m_{e} \dot{v}_{e}=F_{e}$ equation causes an impulse force, $F_{e}$, and corresponding pressure $p_{e}$.

This pressure impulse will always cause the relief valve to open because of its infinite maguitude, no matter how small the $v_{e}^{+}-v_{e}$ difference. The more detailed model of the cylinder includes small elasticity and dissipation parameters, and they are employed to compute a more realistic value of the maximum pressure generated. This can be included in the reduced order model by replacing the $m_{e} \dot{v}_{e}=F_{e}$ equation with the algebraic constraint $K_{e}\left(v_{e}^{+}-v_{e}\right)$ providing the value for $F_{e} . K_{\mathrm{c}}$ is a damping coefficient that captures the $R_{c} C_{c}$ effect. Using this first order approximation, the pressure buildup can be described as $p_{c}^{+}=A_{p} K_{c}\left(v_{e}^{+}-v_{e}\right)$,

If the value of $p_{c}^{+}$exceeds the critical value, $p_{t h}$, this causes a further discontinuous mode change in the relief valve, that goes from closed $\left(\alpha_{0}\right)$ to open $\left(\alpha_{1}\right)$. In this case, the abrupt change in elevator velocity is governed 
by a restitution coefficient, $\lambda_{r}$, defined by the complex ODE model of the relief valve that can be derived in a manner similar to the derivation for the spool valve. The final elevator velocity after the mode transitions is now given by $v_{e}{ }^{+}=\lambda_{r} v_{e}$. The simplified ODE model for $v_{e}$ in the supply mode with relief valve open can be derived similarly.

\section{The Actuator Hybrid Automata}

If the relief valve opens, the effect of $\lambda_{s}$ on the elevator velocity is replaced by $\lambda_{r}$, and, therefore, the change of velocity as computed by $\lambda_{\theta}$ has to be reversed. A hybrid automata that correctly includes this effect is shown in Fig. 7. The modes are $\alpha_{i j}$, where the subscript $i$, represents the mode of the spool valve (2 open, 1 - closed, and 0 - loading), and subscript $j$ represents the mode of the relief valve ( 1 - open, and 0 closed). The corresponding sODEs are also subscripted accordingly. Initially, the actuator is in mode $\alpha_{20}$. In the simplified hybrid automata, the detailed continuous behavior around $x=0$ is abstracted away, and the corresponding discrete events, $\left\{\sigma_{\text {close }}, \sigma_{\text {spool }}, \sigma_{\text {load }}, \sigma_{\text {relief }}\right\}$ are generated by monitoring physical variables. The stroked transitions in Fig. 7 represent transitions where the event generation function, $\gamma$, is applied after the state vector has been updated.

When in $\alpha_{20}$, closing the spool valve moves the system into $\alpha_{10}$ and causes an instantaneous change in the oil flow rate to 0 . Therefore, $q_{s}^{+} \neq q_{s}$ and a rapid drop in the elevator velocity, $v_{e}{ }^{+}=\lambda_{s} v_{e}$, occurs before the valve opens again and goes into the loading mode, $\alpha_{00}$. However, the change in velocity causes a pressure transient, $p^{+}=A_{p} K_{c}\left(v_{e}^{+}-v_{e}\right)$, and if $p^{+}>p_{t h}, \sigma_{\text {relicf }}$ is generated causing the relief valve to open, and the systen goes into mode $\alpha_{11}$, with $v_{e}^{+}=\lambda_{r} v_{\varepsilon}$. Therefore, $v_{\mathrm{e}}{ }^{+}=\lambda_{s} v_{\mathrm{e}}$ is not executed and $v_{\mathrm{e}}^{+}$not affected by mode $\alpha_{10}$. Once the state vector is updated, $q_{s}{ }^{+}=q_{s}$, and $\sigma_{\text {load }}$ is generated causing the system to go into $\alpha_{01}$. If $\sigma_{\text {relief }}$ did not occur, $v_{e}^{+}=\lambda_{s} v_{e}$ remains valid, and after the state vector is updated $q_{s}{ }^{+}=q_{s}$ and the mode transition to $\alpha_{00}$ occurs based on the event $\sigma_{\text {load }}$.

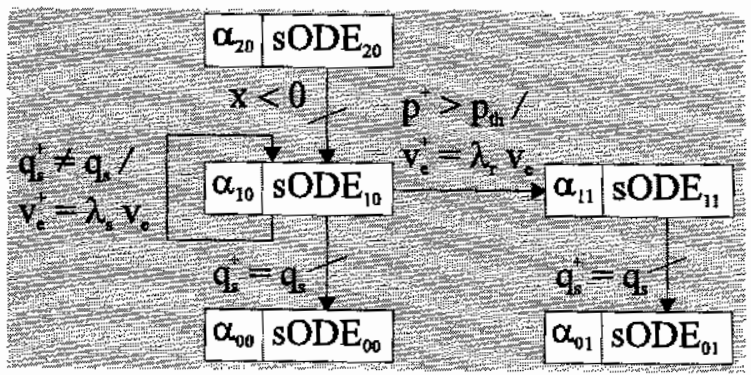

\section{Conclusions}

In this paper we have developed a systematic methodology derived from the singular perturbations approach for generating simpler ODE models for hybrid systems by applying time scale and parameter abstractions to complex nonlinear system models that exhibit fast transient behavior. Compiling the sODEs and transition conditions into hybrid automata with extended execution semantics generates runtime models that can be applied to real-time simulation and analysis of system behavior.

The apparent drawback of creating piecewise simpler hybrid models is that the compositionality property is lost when interactions between the component subsystems have to be analyzed in advance to build the hybrid automata.

\section{References}

[1] R. Alur, C. Courcoubetis, T. A. Henzinger, and P.-H. Ho. Hybrid automata: An algorithmic approach to the specification and verification of hybrid systems. In Lecture Notes in Computer Science, vol. 736, pp. 209-229. Springer-Verlag, 1993.

[2] R. M. Brach. Mechanical Impact Dynamics. John Wiley and Sons, New York, 1991.

[3] W. L. Green. Aircraft Hydraulic Systems. John Wiley, Chichester, UK, 1985.

[4] P. V. Kokotović, H. K. Khalil, and J. O'Reilly. Singular Perturbation Methods in Control: Analysis and Design. Academic Press, London, 1986.

[5] B. Lennartson, M. Tittus, B. Egardt, and S. Pettersson. Hybrid systems in process control. IEEE Control Systems, pp. 45-56, Oct. 1996.

[6] P. J. Mosterman and G. Biswas. A theory of discontinuities in dynamic physical systems. Journal of the Franklin Institute, 335B(3):401-439, Jan. 1998.

[7] P. J. Mosterman and G. Biswas. Building Hybrid Observers for Complex Dynamic Systems using Model Abstractions. In Hybrid Systems: Computation and Control, vol. 1569, pp. 178-192, 1999.

[8] P. J. Mosterman, G. Biswas, and J. Sztipanovits. A hybrid modeling and verification paradigm for embedded control systems. Control Engineering Practice, (6):511-521, 1998.

[9] J. Seebeck. Modellierung der Redundanzverwaltung von Flugzeugen am Beispiel des ATD durch Petrinetze und Umsetzung der Schaltlogik in C-Code zur Simulationssteuerung. Diplomarbeit, Technische Universität Hamburg-Harburg, 1998.

Figure 7: Hybrid automata of an actuator. 